\title{
Smartphone-based drive-test methodology for the experimental characterisation of the physical layer and a performance assessment of LTE networks in urban environments
}

\author{
Stefano Avallone ${ }^{1}$, Nicola Pasquino ${ }^{1}$, Giorgio Ventre ${ }^{1}$, Stefania Zinno ${ }^{1}$ \\ ${ }^{1}$ Dipartimento di Ingegneria Elettrica e delle Tecnologie dell'Informazione, Università degli Studi di Napoli Federico II, Via Claudio 21,80125 \\ Napoli, Italy
}

\begin{abstract}
Long-term evolution (LTE) architecture has recently been enhanced by means of the introduction of multi-antenna systems that make use of multiple input multiple output (MIMO) technology, both at the base transceiver station (eNodeB) and user equipment (UE). MIMO can deploy transmission diversity to achieve more robust transmission in poor propagation conditions and spatial multiplexing to increase throughput and thus LTE performance from an end-user perspective. The most recent version of the standard (Release 10) makes up to eight antennae available for the downlink transmission channel. The paper focuses on the most relevant physical-layer parameters used by LTE to optimise a MIMO system. Performance at different frequencies and bandwidths is investigated over a wide urban area in order to study the network behaviour in actual propagation conditions and to verify whether standard prescriptions are satisfied by a fully operational LTE mobile communication system.
\end{abstract}

\section{Section: RESEARCH PAPER}

Keywords: Measurements; MIMO; LTE physical layer; LTE quality indicators; 4G; multivariate statistical analysis; experimental characterisation

Citation: Stefano Avallone, Nicola Pasquino, Giorgio Ventre, Stefania Zinno, Smartphone-based drive-test methodology for the experimental characterisation of the physical layer and a performance assessment of LTE networks in urban environments, Acta IMEKO, vol. 8, no. 2, article 5, June 2019, identifier: IMEKO-ACTA-08 (2019)-02-05

Section Editor: Emiliano Sisinni, University of Brescia, Italy

Received July 15, 2018; In final form June 11, 2019; Published June 2019

Copyright: This is an open-access article distributed under the terms of the Creative Commons Attribution 3.0 License, which permits unrestricted use, distribution, and reproduction in any medium, provided the original author and source are credited.

Corresponding author: Stefania Zinno; e-mail: stefania.zinno@unina.it

\section{INTRODUCTION}

Multiple input multiple output (MIMO) technology has introduced a strong increase in performance among mobile systems. Contemporary use of multiple antennae at both the transmitter and receiver sides of a MIMO system [1] enables mobile radio systems to achieve substantial performance improvement in terms of transmission efficiency and power consumption. This improvement is thanks to a reliable procedure that provides countermeasures to poor transmissionpath conditions and give the system a broad spectral efficiency. However, MIMO is an even broader technology than that, with multiple implementation starting from its basic principle [2].

The long-term evolution (LTE) standard supports a great variety of MIMO operating schemes suitable for different radio scenarios, and it is able to select schemes dynamically in order to properly address the relevant circumstances. Spatial multiplexing $(\mathrm{SM})$ is expected to give better robustness than transmit diversity (TD). In fact, LTE SM-MIMO in indoor environments, outperforms TD-MIMO and single input multiple output systems because a single data stream transmitted by different antennae can overcome the adverse multipath propagation that is typical of such environments [3]. Furthermore, in [4], the re-enactments demonstrate that the adoption of MIMO is superior to the single input single output approach. In [5], a timeline from Release 8 to the latest Releases of multi-user MIMO (MU-MIMO) techniques is presented. The discussion therein deals with specific aspects of MIMO implementations and related solutions for MU-MIMO. In order to widely investigate LTE and its MIMO implementation, in [6], an eNodeB is tested in a reverberation chamber, and an experimental campaign is carried out to investigate how power boosting affects eNodeB performance in a multipath environment like wireless and vehicular communications. Channel quality parameters are measured, ranging from the 

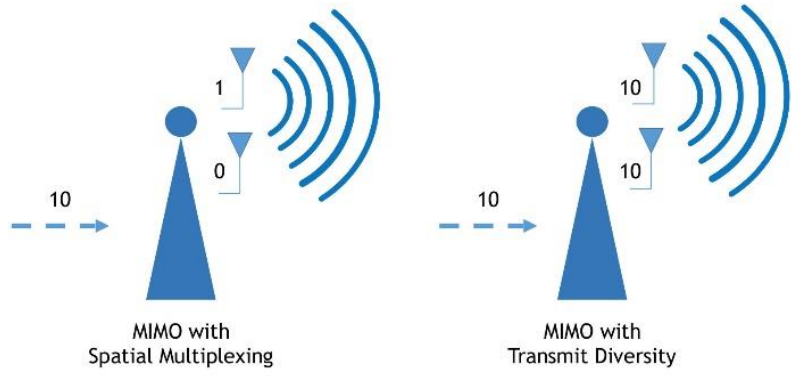

Figure 1. SM and TD MIMO.

empty chamber condition with a highly rich multipath channel to a fully loaded condition in order to mitigate multipath. Anechoic chamber testing is performed in [7]. MIMO user equipment (UE) conditions, and performance is tested along with the implementation of plane-wave synthesis and prefading signal synthesis techniques.

Frequency domain packet scheduling (FDPS) has a great influence on LTE systems [8]. Considering how hard it is to optimise the system, the easiest solution could be to efficiently assign time-frequency resources to UEs by using a proportional fairness metric. One of the first proposals to fully address an LTE MIMO fair comparison of different MIMO schemes is carried out in [9]. The analysis is performed with multiple spatial transmission and a unique signal-and-interference-tonoise ratio (SINR), both with the effect of packet scheduling included in the post-scheduling SINR. The results show that spectral efficiency can benefit significantly from the combined use of SM and FDPS.

In [10]'s experimental setup, an LTE eNodeB shows that the throughput at the lower layers can easily be computed if the preferred channel quality indicator (CQI) and a number of assigned resource blocks (RBs) are well known in advance.

By means of simulations, Sridhar et al. [11] confirm that a channel quality estimation with an inter carrier interference (ICI) cancellation technique reduces complexity and execution time in MIMO networks. At first, the adoption of a maximum likelihood detection scheme is used to suppress the interfering channels, and parallel interference cancellation method and decision statistical combining are adopted in order to cancel ICI and improve data symbol detection.

The estimation process for interference gives rise to a significant criticality in LTE systems that use MU-MIMO. [12] uses a framework for estimation together with a practical solution based on a suboptimal non-iterative method. The nonideal behaviour of MU-MIMO precoding schemes due to poor channel knowledge is also investigated, and a few benefits are presented along with a proposed solution. In [13], several antennae are placed in a 2D array configuration in order to realise spatially separated transmission links to many mobile stations that adopt FD-MIMO. The design and implementation of the 2D antenna array are also presented in detail therein.

The authors of this current article have also contributed to the analysis of LTE network performance using statistical methods in [14]. The aim of the research is to assess network performance when a fully operating MIMO system is adopted, and to compare them to what is stated in the 3GPP standard. Focusing on the downlink channel throughput, measurements are conducted with reference to key parameters for radio optimisation, like SINR assessed directly on the end-user side.
The results of an extensive measurement campaign and system characterisation highlight that standard expectations are too high with respect to actual urban environment deployment of the system.

The paper is organised as follows: In Section 2, we describe MIMO features and their implementation. In Section 3, the experimental setup is presented, while results are discussed in Section 4. Finally, Section 5 will then draw conclusions and present some considerations about ongoing research.

\section{MIMO: IMPROVING EFFICIENCY AND THROUGHPUT}

Multi-antennae technologies for reception and transmission at both the eNodeB and UE side have enabled high LTE performance.

MIMO antennae can bring several potential benefits to mobile radio systems, including more reliable execution of system procedures under poor signal conditions, wider spectral efficiency, and increased data rates for mobile users. Although higher performance can be achieved by resorting to multiple antennae and SM techniques, systems costs are higher when compared to single antenna-based systems, due to an increase in hardware costs and complexity. MIMO systems also reduce the effects of fading in the propagation channel at the cost of advanced software requirements. Since Release 10 (also known as LTE-advanced [15]), up to eight antennae are available for the downlink channel. At a basic level, however, the goal is to ensure an error rate of $10 \%$ or fewer, allowing the UE to take advantage of the SM.

\subsection{Configurations}

MIMO in LTE can adopt the $2 \times 2,4 \times 4$, and $8 \times 8$ antenna configurations. It can also be configured, as mentioned above, in two modes, SM and TD, as shown in Figure 1. On the one hand, the aim of SM is to transmit multiple data streams with different content over different parallel channels in order to increase the data rate. It takes advantage of multipath propagation to create several independent transmission channels between the transmitter and receiver, which enables two or more different signal streams to be transmitted simultaneously. Using proper coding and signal processing, information on the different streams can be extracted independently at the receiver side. This technique has led to an increase of the throughput available to a single user or allows multiplex data from multiple users. The maximum number of channels that can be multiplexed together is equal to the smaller of the number of antennae on both the transmitter and receiver sides. On the other hand, if a single user is taking advantage of a single information flow, then TD can achieve spatial diversity performance with the use of multiple antennae at the transmitter side. Several versions of the same information stream are sent to the receiver as a countermeasure to channel fading and to improve SINR. TD techniques are adopted when a UE is experimenting the worst channel conditions.

Both closed loop and open loop are implemented in the LTE spatial diversity operating modes. With open loop, spatial diversity is adopted blindly, while with the use of closed loop, control information is collected by the UEs in order to properly weight the antennae.

When SU-MIMO is adopted, the basic idea is to transmit a multiple codeword to a single user in the same time-frequency interval so that there is only one scheduled user per sub-band or channel. A codeword is defined as a coded transport block, but in a wider sense, we consider it as a single independent data 
Table 1. TM features and main quantities reported.

\begin{tabular}{|c|c|c|}
\hline $\begin{array}{l}\text { Transmission } \\
\text { mode }\end{array}$ & Features & Main quantities reported \\
\hline TM1 & $\begin{array}{l}\text { Single transmitting } \\
\text { antenna }\end{array}$ & $\begin{array}{l}\text { UE-selected sub-band and wide- } \\
\text { band CQI; } \\
\text { Higher-layer configured wide-band } \\
\text { and sub-band CQI, no PMI. }\end{array}$ \\
\hline TM2 & Transmit diversity & $\begin{array}{l}\text { UE-selected sub-band and wide- } \\
\text { band CQI; } \\
\text { Higher-layer configured wide-band } \\
\text { and sub-band CQI, no PMI. }\end{array}$ \\
\hline TM3 & $\begin{array}{l}\text { Open loop SM with } \\
\text { cyclic delay } \\
\text { diversity (CDD) }\end{array}$ & $\begin{array}{l}\text { UE-selected sub-band and wide- } \\
\text { band CQI; } \\
\text { Higher-layer configured wide-band } \\
\text { and sub-band CQI, no PMI. }\end{array}$ \\
\hline TM4 & $\begin{array}{l}\text { Closed loop SM } \\
\text { (feedback from the } \\
\text { UE) }\end{array}$ & $\begin{array}{l}\text { Wideband CQI per codeword and } \\
\text { PMI for each sub-band; } \\
\text { UE-selected sub-band and wide- } \\
\text { band CQI per codeword, and PMI; } \\
\text { Higher-layer configured wideband } \\
\text { and sub-band CQI, and PMI. }\end{array}$ \\
\hline
\end{tabular}

stream. Although a MU-MIMO system can perform much better than a SU-MIMO one, we only focus on SU-MIMO in the following sections, because that is the system configuration adopted in our experimental campaign.

\subsection{Transmission modes}

In the downlink, LTE, apart from MIMO, offers fall-back technologies such as TD or SISO. It also supports different transmission modes (TMs) for each individual UE. Which mode is applied is determined by both the capability of the UE and the capabilities and engineering of the eNodeB. The TM specifies how to process data transmissions.

For example, SISO is implemented in TM1, while TD is identified with TM2. SM is introduced in TM3 and TM4, which implement open loop and closed loop transmission, respectively; the former applies blind spatial diversity, and the latter uses data collected by the UE to properly weight each antenna. Good summaries of each TM can be found in Table 7.2.3-0 in [16] and in Table 1.

When TM1 is in use, the system transmits one single layer from each antenna port. Reference signals (RSs) combined with data are transmitted, adopting the same antenna configuration. To demodulate the signal at the receiver side, only the RS related to that specific user is required. Although many antennae are simultaneously transmitting, with this technique, a single UE is not aware of the actual number of parallel transmission and perceives data as it was transmitted over the single stream.

To improve SINR and robustness of transmission, TM2 implements a classical MIMO. All data is in fact, sent via multiple antennae equipped with different modulation schemes and frequency configurations. When TM2 is in use, only a single layer is mapped with a space frequency block code (SFBC) that relies on the Alamouti code [17] and is transmitted from multiple antennae. When two antennas are adopted, a frequency-based version of SFBC is used, while with a combination of four antennae, a mix of SFBC and frequency switched transmit diversity is used.

In the case of a UE moving at high speed, so when channel information is missing or when the channel rapidly changes, SM is implemented with TM3.

When TM4 is implemented, each transmission is mapped to one or more layers. TM4 adopts a closed loop technique, which grants channel estimation at the receiver.

\subsection{CQI reporting}

In LTE networks, UEs usually estimate channel state information and aggregate it into a channel status information (CSI) report, simply analysing the network data retrieved from the physical layer measurement. CSI comprises [16]:

- CQI;

- Precoding matrix indicator (PMI);

- Precoding type indicator (PTI); and

- Rank indication (RI).

Measurements should be conducted to determine the optimal CQIs under different CSI conditions.

To properly tune modulation and coding scheme (MCS), UEs are used to periodically transmit CSI to the eNodeB in the form of a CQI report, based on the measurements that are periodically carried out by mobiles. CQI values span from 0 to 15. The higher the CQI, the higher the order of modulation suggested for adoption by the UE. CQI can be related to the overall operating band (wideband CQI) or to a few selected sub-bands of interest (sub-band CQI).

The wideband report provides a CQI value computed by the UE for the whole downlink bandwidth, while in the calculation of sub-band CQIs, it is assumed that each transmission occurs only in the preferred bands. If a UE-selected sub-band feedback is chosen, as in the eNodeB-configured one, both kinds of CQIs are collected i.e. the sub-band CQI is the average value measured in the $M$ selected sub-bands, each of a specific size. Typically, the higher layer configured sub-band report provides the highest granularity [16].

Each TM requests a distinct set of reporting. With TM4, implementing a closed loop technique, the UE sends a full report to the base station as a response about the channel conditions. A full report also deals with PMI configuration, which is periodically sent, and possible pre-coding matrices are shared between the receiver and the transmitter. TM2 and TM3, on the other side, require no feedback at all or less information from the actual user about the channel propagation conditions.

\subsection{Measurement capabilities for E-UTRA}

Different quantities are measured at the physical layer, and in the following section, a brief review of the most significant ones is given.

One key parameter is the Received Signal Strength Indicator (RSSI), which is the total received power, thus including both serving and non-serving co-channel cells interference, adjacent channel interference, thermal noise and the noise generated within the receiving demodulator bandwidth.

With reference to a cell-specific RS, SINR is defined as the linear average of the power contribution given by the resource element (RE) divided by the linear average of the sum of noise and interference power within the same frequency bandwidth.

Then, for a specific cell, the reference signal received power (RSRP) is defined as the average power of the portion of frame 


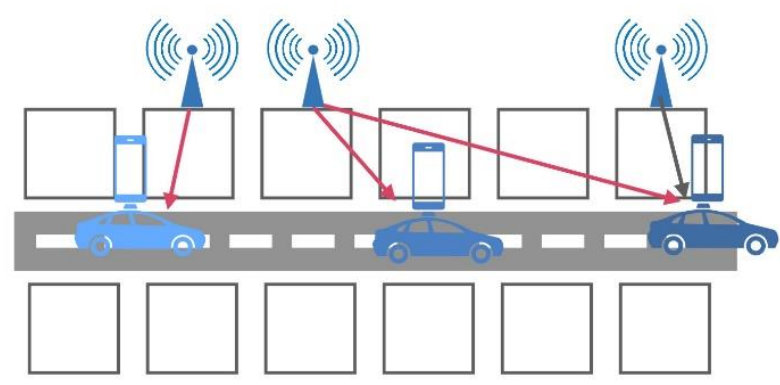

Figure 2. Drive-test measurement campaign concept.

that carries cell-specific RS within the considered bandwidth. Expressed in $\mathrm{dBm}$, it is calculated as:

$\mathrm{RSRP}=\mathrm{RSSI}-10 \log (12 \cdot N)$,

where $N$ is the number of RBs of the LTE's RSSI measurement bandwidth.

Reference signal received quality (RSRQ) is a cell-specific signal quality metric. It is defined in linear units as:

$R S R Q=N \frac{\text { RSRP }}{\text { RSSI }}$

Like RSRP, RSRQ is mainly used to provide ranking among different cells as candidates to become the serving cell based on their signal quality, and it represents the quality of the received RS.

\section{EXPERIMENTAL SETUP}

The measurement campaign was conducted in an urban area of a city in southern Italy by means of a drive-test experimental activity, as seen in Figure 2, where the UE is placed inside a car that is driven around the investigated area to collect georeferenced measurements, which are then post-processed. The figure also shows that the serving cell (the one connected by a red arrow) is not always the nearest one; rather, it is the one that has the parameter values that best fit the standard requirements.

Such methodology has been already explored and used in [14], [18], [19], [23], [24]. The location of measurements cannot be indicated due to a non-disclosure agreement signed between the authors of this article and the mobile operator. The only available information is that data was collected over a coastal city, which therefore has both typical urban propagation and clutter signal due to the sea, which is known to affect propagation by surface reflection and may therefore increase interference significantly.

During the measurement campaign, we intentionally did not analyse just one source of traffic. Rather, we monitored a few hours of a typical day of normal smartphone usage (both traffic and voice data were collected), in the time interval between 8 am and $12 \mathrm{am}$, which are typically peak hours of traffic for a cellular network.

The UE has neither a specific hardware or software configuration, nor has it been placed in a specific position

Table 2. System and smartphone configuration.

\begin{tabular}{ccccc}
\hline System & $\begin{array}{c}\text { System } \\
\text { frequency }\end{array}$ & Band & $\begin{array}{c}\text { Samsung } \\
\text { Model }\end{array}$ & $\begin{array}{c}\text { NEMO Handy } \\
\text { Version }\end{array}$ \\
\hline LTE FDD & $800 \mathrm{MHz}$ & 20 & S7-Edge & 2.62 .337 \\
LTE FDD & $2100 \mathrm{MHz}$ & 1 & S5 & 2.62 .337 \\
\hline
\end{tabular}

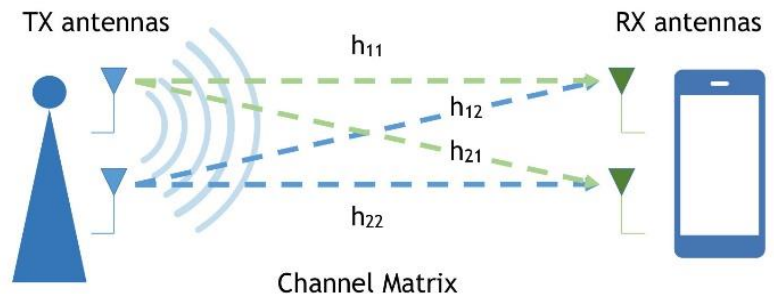

Figure 3. MIMO $2 \times 2$ spatial multiplexing.

within the car so as to mimic the typical position it would have during normal use i.e. it was placed randomly in the driver's jacket pocket, on the passenger seat, or on the dashboard.

Assessment of the network performance was carried out using the Nemo Handy tool. The software provides automated results in a logbook format from UEs' raw measurement data. It acts like a live network monitoring tool, and for each event, it collects different datasets. In our experiment, due to the reduced number of samples from two of the four LTE bands tested, data from only two UEs are reported and analysed, each set at a specific system carrier frequency/bandwidth value of an LTE frequency division duplex (FDD) system, namely:

- LTE FDD 2100 band 1 (10 MHz bandwidth);

- LTE FDD 800 band 20 (20 MHz bandwidth).

Hardware and software specifications are summarised in Table 2. However, other system carrier frequencies have been investigated but are not discussed in the paper due to the lack of samples collected.

The network under test implements a $2 \times 2$ MIMO, as shown in Figure 3. We refer the reader to the Nemo Analyzer specifications and features [20] for specific information on each event that the software can monitor. We limit our focus to the content provided for LTE technology regarding CellMeas (shown in Table 3), CQI, and carrier per interference (CI) events. The events and the related parameter values were monitored according to the 3GPP Technical Specification for Evolved UMTS Terrestrial Random-Access Network (eUTRAN) version 12 [21] (see Section 2.4 for an explanation of the physical layer quantities), and measurements were recorded when samples of interests are received. CellMeas stores RSSI in $\mathrm{dBm}, \mathrm{RSRP}$ between 0 and $140 \mathrm{dBm}$, RSRQ between 0 and 30 $\mathrm{dB}$; CQI explores: wideband and sub-band CQI for both Codeword 0 and 1 , in the discrete range between 0 and 15 . CI

Table 3. Nemo Handy CellMeas Event

EVENT ID CELL MEAS

Cellular System

GSM, TETRA, UMTS FDD, IDEN, UMTS TD-SCDMA, LTE FDD, LTE TDD, cdmaOne, CDMA 1x, EVDO, WLAN, GAN, WLAN, WIMAX, AMPS, DAMPS, NAMPS

Record State

Always

Recordings occur when the retrieved sample is -50 received from the UE. It is not mandatory that -100 all received samples are recorded and the current frequency of recording is about twice per second when the UE is in a connected state. Separate measurement event logging is kept for each system.

Tool Nemo 


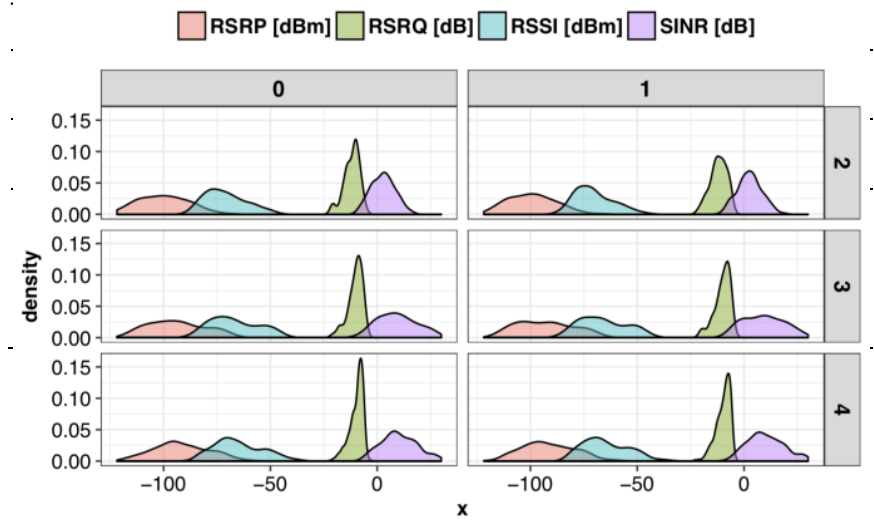

Figure 4. RS-related epdf for each TM and Codeword for Band 20 (800 $\mathrm{MHz}$ ).

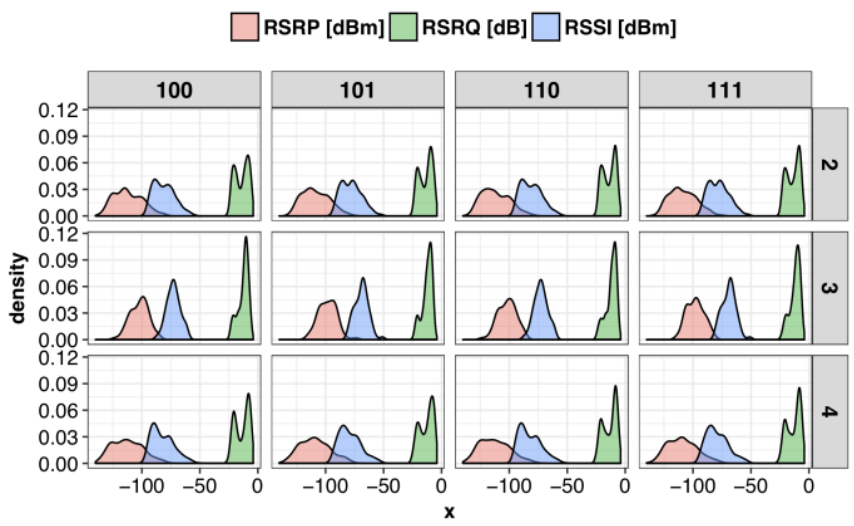

Figure 5. RS-related epdf for each TM and Codeword for Band 1 (2100 $\mathrm{MHz}$ ).

investigates RS SINR, whose calculation method can depend on the device hardware. It can reach up to $50 \mathrm{~dB}$.

\section{MIMO ANALYSIS}

In this section, we present a MIMO analysis of the supervised operator. Most of the time, both systems adopt TM4 with closed loop SM and TM2 with TD. As already stated, TM4 is in use when channel conditions are favourable, so we expect that the physical layer parameters confirm that we are in good channel propagation condition.

\subsection{Physical layer quantities statistical analysis}

The experimental probability density function (epdf) corresponding to the physical layer parameters presented in Section 2 are shown in Figure 4 and Figure 5 with respect to the different TMs and codewords. For Band 20, only the values measured at TX (codeword 0,1 ) are collected, while for Band 1, values are collected for both TXs and RXs.

For Band 20 (Figure 4), channel conditions are shown to be in a good range, while for Band 1 (Figure 5), we can clearly see a worsening of the RSRQ values, possibly due to the poor propagation properties of the higher frequencies. The SINR pdf is missing in Figure 5 due to a lack of samples.

There also seems not to be much of a difference between TMs and codewords, as shown in Figure 4, besides a slight and statistically insignificant shift to the left in the SINR density peak value position and a significantly smaller variance value for TM2, unlike the expected behaviour. This finding can be explained by that mode, which is based on TD, being
RSRP [dBm] $\odot R S R Q[d B] \odot R S S I[d B m] \odot \operatorname{SINR}[d B]$

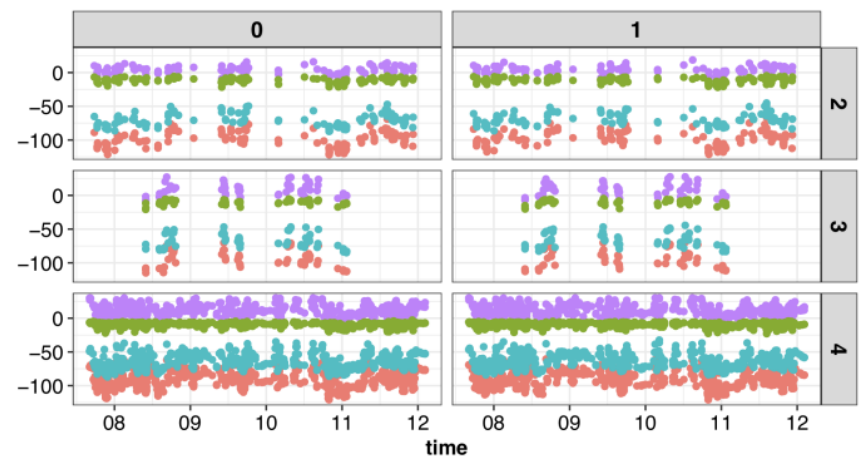

Figure 6. RS-related quantities time samples for Band $20(800 \mathrm{MHz})$.

- RSRP [dBm] $\odot \mathrm{RSRQ}[\mathrm{dB}] \odot \mathrm{RSSI}[\mathrm{dBm}] \odot \operatorname{SINR}[\mathrm{dB}]$

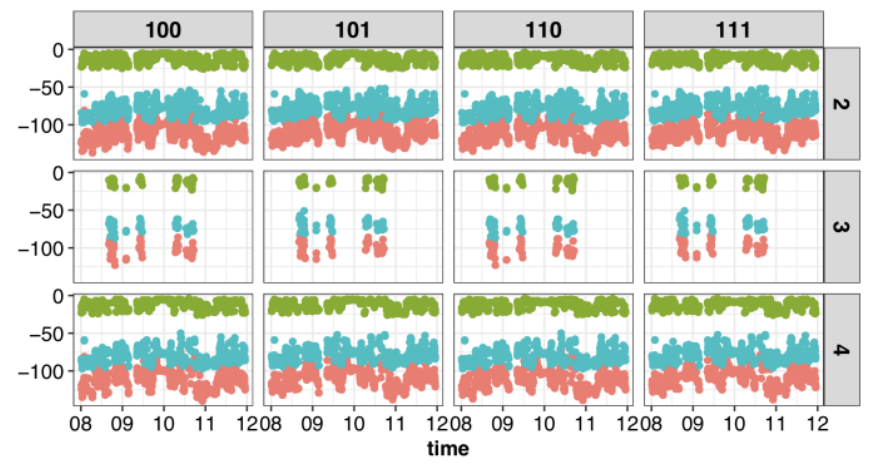

Figure 7. RS-related quantities time samples for Band $1(2100 \mathrm{MHz}$ ).

specifically designed to enhance network performance in terms of SINR. In addition, it is apparent from the picture that RSRQ, unlike the other quantities, has a smaller variance i.e. it is more stable in its mean, leading to the RS's overall more stable quality. Furthermore, the significant difference between RSSI and RSRP, the former being about $25 \mathrm{~dB}$ greater than the latter, shows that the network is affected by a large interfering signal.

In Figure 5, we can see that TM3 differs from the other two modes. As a matter of fact, while RSRQ presents a less prominent bimodality, RSRP and RSSI are slightly shifted to larger values, yet with a smaller variance and almost monomodal behaviour (less so for RSRP).

In Figure 6 and Figure 7, the time samples of RSRP, RSSI, RSRQ, and SINR are shown. For both systems, when TM3 is in use, we can see fewer samples, matching that TM's standard specifications. TM3 implements open-loop SM and is adopted when few retrievals by the user are required in order for the system to work (poor channel conditions). On the other hand, we can see almost continuous reporting of values when TM4 and TM2 are adopted. Again, this fully respects the standard specifications, since TM4 implements closed-loop multiplexing, and TM2 respects standard expectations to inform the network about channel conditions.

In accordance to Figure 4 and Figure 5, we can see that RSRP and RSSI differ by a few tens of $\mathrm{dBs}$, showing quite a large interference, possibly due to co-channel contributions caused by an unexpected larger area covered by the involved base stations as an effect of sea water, which acts like a reflector.

The numbers of samples for each TM are compared in 
Table 4, where (reading from left to right) the total time of use and total number of samples of each system as well as the number of samples in each mode (together with the corresponding percentage over the total number of samples) are reported. Values are clearly in accordance with what is graphically perceived from Figure 6 and Figure 7.

\subsection{CQI report analysis}

In Figure 8, the UE CQI samples are shown for Band 20. In accordance with TM implementation and requirements, both sub-band CQI and wideband CQI are reported. Samples are fewer and with much lower values for TM3, since UE adopts this TM rarely, and the report is richer for codeword 0 . With TM4, the samples are heavily collected, mostly for wideband CQI, while with TM2, implementing TD, sub-band CQI has a predominant role.

In Figure 9, referring to Band 1, a massive wideband CQI report takes place, while no sub-band CQI samples are reported. Even if for codeword 1 we recorded fewer samples (no one at all for TM2), we can appreciate a different behaviour in TM3, where lower variance wideband CQI samples are reported, and in TM4, where higher values are collected.

We reiterate that when CQI values are higher, higher order modulations are suggested for use for UE transmissions. Comparing the physical layer parameters shown in Figure 6 and Figure 7 with the related CQI values shown in Figure 8 and Figure 9, with better channel conditions, higher modulation orders are suggested through CQIs, and the reporting performs as depicted in 3GPP Standard-as we expected.

We also stress that each vendor operates differently to calculate CQI, and 3GPP does not mandate how to compute it. In this experiment, however, if the eNodeB follows the UE's

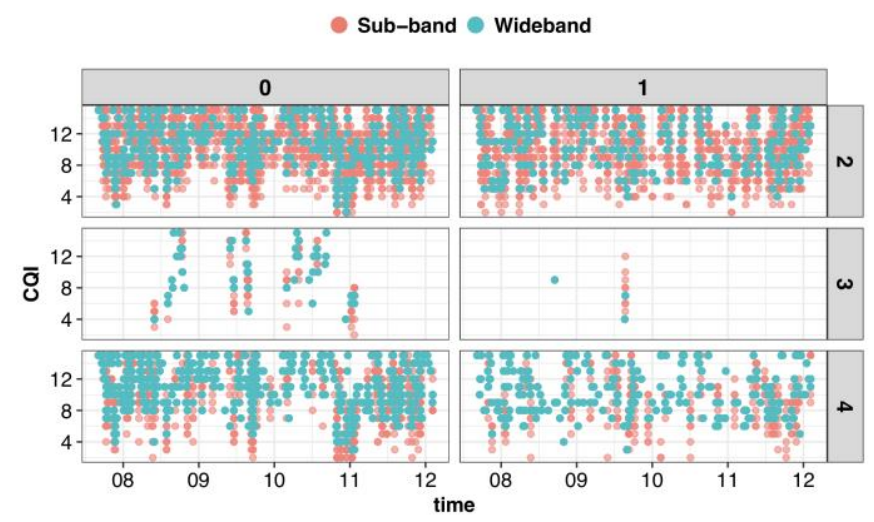

Figure 8. CQI for each TM and Codeword for Band $20(800 \mathrm{MHz})$.

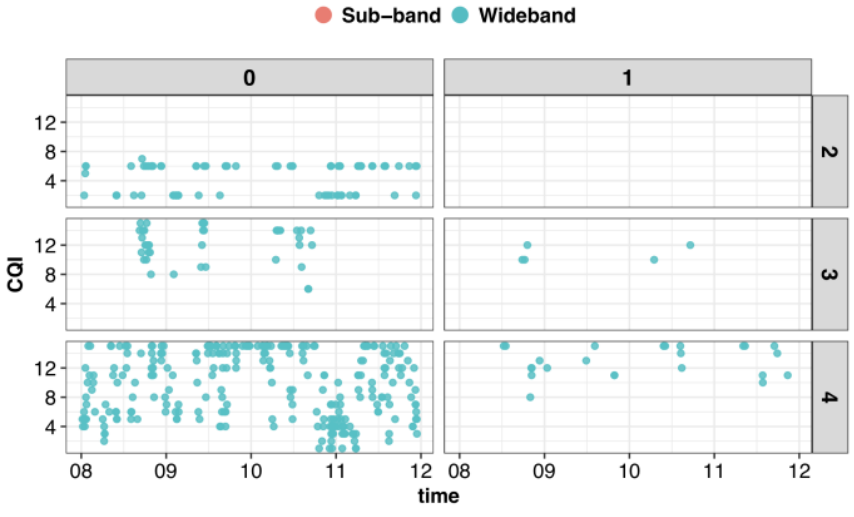

Figure 9. CQI for each TM and Codeword for Band 1 (2100 MHz). indications without overriding them, based on the outcome of both this section and the previous one, CQI appears to be computed on RSRQ and SINR quantities. This indication could be truthful since, as stated, RSRQ calculation indicates the overall quality of the received reference signal, and it seems to be the best parameter for calculating CQI.

\section{CONCLUSIONS}

In this paper, we provided the results of a smartphone-based experimental characterisation of LTE network MIMO performance in an urban environment through a drive-test methodology. With reference to the investigated physical layer parameters, it is evidenced that the LTE architecture performs as expected by the relevant standard, although it is necessary to investigate the relationship between those parameters and some user-related ones, such as the network throughput.

For each TM, we highlight that the expected behaviour is generally encountered and that the $3 \mathrm{GPP}$ requirements are mostly matched, despite the complex propagation scenario.

The analysis of the physical layers quantities has led us to the conclusion that the evolution over time of RSRP, RSSI, RSRQ, and SINR is efficiently reported, respecting standard expectations to inform the network about channel conditions.

TM4 reports all CQI mandatory parameters together with TM2. Additionally, TM3 reports a dataset even if the standard does not mandate reporting any sort of information to eNodeBs.

With respect to CQI reporting for different TMs, the UEselected sub-band and wideband CQI are shown. With the information provided by physical layer quantities and with favourable channel conditions, higher order modulation is suggested through CQIs, and the reporting performs as envisioned in the 3GPP standard-as we expected.

Finally, this paper provides a certainly prodromal and possibly mandatory analysis for future thorough investigation of the quantities in which the end-user is mostly interested, like bit (or block) error rate and throughput in the downlink channel. By the joint study of such end-user quantities and physical layer ones, it will be possible to determine network criticalities and, where possible, to actuate corrective action for better network performance.

\section{REFERENCES}

[1] S.Sesia, I.Toufik, M.Baker, LTE - The UMTS Long Term Evolution: From Theory to Practice. $2^{\text {nd }}$ ed., John Wiley \& Sons, Chichester, UK, 2011, ISBN 978-0-470-66025-6.

[2] A.Benjebbour, A.Li, K.Takeda, Y.Kishiyama, T.Nakamura, Y.Inoue, Y.Kishiyama, T.Nakamura, 'Advanced Multiple-access and MIMO Techniques’, in: Toward 5G Appl. Requir. Candidate Technol., R.Vannithamby, S.Talwar (editors). John Wiley \& Sons, Chichester, UK, 2016, pp. 222-249, ISBN 978-1-11897983-9.

[3] D.Nguyen-Thanh, T.Le-Tien, C.Bui-Thu, T.Le-Thanh, 'LTE indoor MIMO performances field measurements', Proc. of the 2014 Int. Conf. Adv. Technol. Commun. (ATC 2014) IEEE, Oct, 2014, pp. 84-89.

[4] T. PadmaPriya, V.Saminadan, Performance improvement in long term evolution-advanced network using multiple input multiple output technique, J. Adv. Res. Dyn. Control Syst., 9(6) (2017) pp. 990-1010.

[5] C.Lim, T.Yoo, B.Clerckx, B.Lee, B.Shim, Recent trend of multiuser MIMO in LTE-Advanced, IEEE Communications Magazine, 51(3) (2013) pp. 127-135. 
[6] D.Micheli, M.Barazzetta, F.Moglie, V.M.Primiani, Power boosting and compensation during OTA testing of a real 4G LTE base station in reverberation chamber, IEEE Transactions on Electromagnetic Compatibility, 57(4) (2015) pp. 623-634.

[7] M.S.Miah, D.Anin, A.Khatun, K.Haneda, L.Hentila, E.T.Salonen, On the field emulation techniques in over-the-air testing: experimental throughput comparison, IEEE Antennas Wirel. Propag. Lett., 16 (2017) pp. 2224-2227.

[8] Q.ul Ain, S.R.ul Hassnain, M.Shah, S.A.Mahmud, 'An evaluation of scheduling algorithms in LTE based 4G networks', Proc. of the 2015 International Conference on Emerging Technologies (ICET), Dec, 2015, Peshawar, Pakistan, pp. 1-6.

[9] N.Wei, A.Pokhariyal, T.B.Sørensen, T.E.Kolding, P.E.Mogensen, Performance of spatial division multiplexing MIMO with frequency domain packet scheduling: from theory to practice, IEEE Journal on Selected Areas in Communications, 26(6) (2008) pp. 890- 900

[10] M.Slanina, L.Klozar, S.Hanus, 'Practical measurement of data throughput in LTE network depending on physical layer parameters', Proc. of the 2014 24th IEEE Int. Conf Radioelektronika, RADIOELEKTRONIKA 2014, Apr., 2014, Bratislava, Slovakia, pp. 1-4.

[11] P. Sridhar, M.R.Sumalatha, 'Interference cancellation and channel estimation for MIMO-LTE-A networks', Proc. of the 2016 International Conference on Wireless Communications, Signal Processing and Networking (WiSPNET), Mar., 2016, Chennai, India, pp. 2098-2103.

[12] R.A.Abdelaal, A.S.Behbahani, A.M.Eltawil, 'Practical framework for downlink MU-MIMO for LTE systems', Proc. of the IEEE Wireless Communications Letters, 6(3) (2017) pp. 314-317.

[13] Y.Kim, H.Ji, J.Lee, Y.H.Nam, B.L.Ng, I.Tzanidis, Y.Li, J.Zhang, Full dimension MIMO (FD-MIMO): the next evolution of MIMO in LTE systems, IEEE Wireless Communications, 21(3) (2014) pp. 92-100.

[14] S.Avallone, N.Pasquino, S.Zinno, D.Casillo, 'Smartphone-based measurements of LTE network performance', Proc. of the 2017 IEEE Int. Instrum. Meas. Technol. Conf., May, 2017, Torino, Italy, pp. 1-6, doi: 10.1109/I2MTC.2017.7969854

[15] 3gpp TR 36.871 Technical Report 3rd Generation Partnership Project; Technical Specification Group Radio Access Network; Evolved Universal Terrestrial Radio Access (E-UTRA);
Downlink Multiple Input Multiple Output (MIMO) Enhancement for LTE-Advanced.

[16] (2009-10) ETSI TS 136213 v8.8.0 Technical Specification LTE; Evolved Universal Terrestrial Radio Access (E-UTRA); Physical Layer Procedures (3gpp TS 36.213 version 8.8.0 release 8).

[17] ETSI TS 136212 v8.8.0 (2010-01) Technical Specification LTE; Evolved Universal Terrestrial Radio Access (E-UTRA); Multiplexing and Channel Coding (3gpp TS 36.212 version 8.8.0 release 8).

[18] F.Afroz, R.Subramanian, R.Heidary, K.Sandrasegaran, S.Ahmed, SINR, RSRP, RSSI and RSRQ measurements in long term evolution networks, Int. J. Wirel. Mob. Networks, 7(4) (2015) pp. 113-123.

[19] M.B.Albaladejo, D.J.Leith, P.Manzoni, 'Measurement-based modelling of LTE performance in Dublin city', Proc. of the 2016 IEEE 27th Annual International Symposium on Personal, Indoor, and Mobile Radio Communications (PIMRC), Sept., 2016, Valencia, Spain, pp. 1-6.

[20] Nemo Analyzer.

[Online]. Available: https://www.keysight.com/en/pd-2767469pn-NTN00000A $/$ nemo- analyze? $\mathrm{cc}=\mathrm{EN} \& \mathrm{lc}=\mathrm{en}$

[21] 3GPP TS 36.214 v12.2.0 (2015-03) Technical Specification 3rd Generation Partnership Project; Technical Specification Group Radio Access Network; Evolved Universal Terrestrial Radio Access (E-UTRA); Physical Layer; Measurements (release 12).

[22] (2012-10) LTE; Evolved Universal Terrestrial Radio Access (EUTRA); User Equipment (UE) Conformance Specification; Radio Transmission and Reception; Part 1: Conformance Testing (3gpp TS 36.521-1 version 10.3.0 release 10).

[23] S.Avallone, N.Pasquino, S.Zinno, D.Casillo, 'Experimental characterization of LTE adaptive modulation and coding scheme under actual operating conditions', Proc. of the 2017 IEEE International Workshop on Measurement and Networking (M\&N), 2017, Naples, Italy, pp. 1-6. doi: 10.1109/IWMN.2017.8078401.

[24] S.Avallone, N.Pasquino, G.Ventre, S.Zinno, 'Experimental characterization of long term evolution multiple input multiple output performance in urban propagation scenarios', Proc. of the 2018 Workshop on Metrology for Industry 4.0 and IoT, 2018, Brescia, Italy, pp. 254-259. doi: 10.1109/METROI4.2018.8428318. 